\title{
The number of metastable states in the generalized random orthogonal model
}

\author{
R. Cherrier, D.S. Dean and A. Lefèvre \\ IRSAMC, Laboratoire de Physique Quantique, Université Paul Sabatier, 118 route de \\ Narbonne, 31062 Toulouse Cedex 04, France \\ E-mail: lefevre@irsamc.ups-tlse.fr
}

\begin{abstract}
We calculate the number of metastable states in the generalized random orthogonal model. The results obtained are verified by exact numerical enumeration for small systems sizes but taking into account finite size effects. These results are compared with those for Hopfield model in order to examine the effect of strict orthonormality of neural network patterns on the number of metastable states.

PACS numbers: $05.20-\mathrm{y}, 75.10 \mathrm{Nr}$
\end{abstract}

\section{Introduction}

Mean field or totally connected spin glass models are among the most widely studied models of complex systems. They are the starting point for understanding finite dimensional spin glasses and are also related to neural network models and complex optimization problems [1]. Such systems exhibit an exponentially large number of pure states and dynamic glass like transitions. Below a certain dynamic transition temperature the dynamics becomes very slow and the systems stay out of equilibrium on numerical or experimental time scales. An important factor in the slow dynamics is the presence of metastable or blocked configurations. The enumeration of the number of metastable states has been addressed by various authors in $p$-spin Ising systems with Gaussian interaction matrices [2], neural network models [3, [4, random orthogonal models (ROMs) [5 and in periodic glass models which have no quenched disorder [6]. Recently the authors studied the statics of generalized random orthogonal models originally introduced in [7] and established general criteria determining whether these models exhibit continuous spin glass like transitions or structural glass transitions preceded by a dynamical transition [8]. The first known examples of these sorts of phase transitions were seen in $p$-spin models for $p>2$ [9]. However a number of two spin Ising models were later shown to have this structural glass transition [7, 8, [1], [1]. Here we shall extend the results of [5] for the ROM to a more general ROM which can be interpreted as a Hopfield model with strictly orthogonal patterns in order to explore the influence of pattern orthogonality on the number of metastable states. We also 
analyze anti-ferromagnetic Hopfield model as this class of ROMs can also be viewed as anti-ferromagnetic Hopfield models. The calculation for the standard ferromagnetic

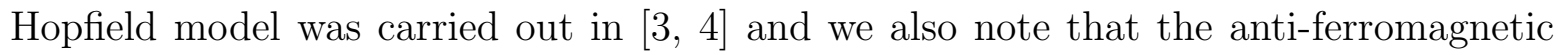
Hopfield has a structure similar to the Hamiltonian arising in the analysis of the Nash equilibria in the minority game as studied in [11]. Our analytic results are backed up by exact enumeration simulations for small system sizes. Despite small system sizes we show that when finite size scaling is taken into account the agreement with the analytical results is excellent.

The Hamiltonian in a fully connected generalized ROM is

$$
H=-\frac{1}{2} \sum_{i j} J_{i j} S_{i} S_{j}
$$

where the $S_{i}, 1 \leq 1 \leq N$ are Ising spins and the interaction matrix $J$ is statistically invariant under the transformation $J \rightarrow \mathcal{O}^{T} J \mathcal{O}$ where $\mathcal{O} \in O(N)$ (the group of orthogonal transformations on $\mathbb{R}^{N}$ ). The matrix $J$ can thus be written as

$$
J=\mathcal{O}^{T} \Lambda \mathcal{O}
$$

where $\Lambda$ is a diagonal matrix with density of eigenvalues denoted by $\rho(\lambda)$. If the support of $\rho(\lambda)$ is bounded on the real axis then the thermodynamic limit is well defined. It was shown in [8] that the nature of the spin glass transition in such models depends on the behavior of the density of states $\rho(\lambda)$ in the neighborhood of $\lambda_{\max }$, the largest eigenvalue of $\Lambda$.

In this paper we shall concentrate on the model defined by

$$
\rho(\lambda)=\alpha \delta(\lambda-1)+(1-\alpha) \delta(\lambda+1)
$$

The matrix $J$ in this case may be written as

$$
\begin{aligned}
J_{i j} & =\sum_{\mu} \lambda_{\mu} \xi_{i}^{\mu} \xi_{j}^{\mu} \\
& =\sum_{\left\{\mu: \lambda_{\mu}=1\right\}} \xi_{i}^{\mu} \xi_{j}^{\mu}-\sum_{\left\{\mu: \lambda_{\mu}=-1\right\}} \xi_{i}^{\mu} \xi_{j}^{\mu}
\end{aligned}
$$

where $\xi^{\mu}$ is a random basis of orthonormal vectors on $\mathbb{R}^{N}$. One may also write $J$ in the following two forms using the completeness of the $\xi^{\mu}$.

$$
\begin{aligned}
J_{i j} & =2 \sum_{\left\{\mu: \lambda_{\mu}=1\right\}} \xi_{i}^{\mu} \xi_{j}^{\mu}-\delta_{i j} \\
& =\delta_{i j}-2 \sum_{\left\{\mu: \lambda_{\mu}=-1\right\}} \xi_{i}^{\mu} \xi_{j}^{\mu}
\end{aligned}
$$

We recall that the Hopfield model with $p=\alpha N$ Gaussian patterns has an interaction matrix given by

$$
J_{i j}=\sum_{\mu=1}^{\alpha N} \xi_{i}^{\mu} \xi_{j}^{\mu}
$$


Here the variables $\xi_{i}^{\mu}$ are Gaussian with zero mean and variance $\overline{\xi_{i}^{\mu} \xi_{j}^{\mu^{\prime}}}=\delta_{i j} \delta^{\mu \mu^{\prime}} / N$. These patterns are only orthonormal in the statistical sense, that is $\sum_{i} \overline{\xi_{i}^{\mu} \xi_{i}^{\mu^{\prime}}}=\delta^{\mu \mu^{\prime}}$. Up to a constant diagonal term, the ROM we study here is from Eq. (5) equivalent to a ferromagnetic Hopfield $(\mathrm{FH})$ model with $\alpha N$ strictly orthonormal patterns, or from Eq. (6) equivalent to an anti-ferromagnetic Hopfield (AFH) model with $1-\alpha$ patterns.

The number of metastable states gives useful information about the phase space of complex systems. The easiest metastable states to analyze are those which are single spin flip stable, that is to say a configuration where flipping a single spin increases (and possibly leaves constant) the energy of the system. Alternatively every spin is aligned with its local field. A metastable state thus defined is a blocked configuration of any single spin flip Monte-Carlo dynamics.

\section{Average Number of Metastable States}

In this section following we explain the calculation of the number of metastable states for generalized ROMs. By definition the average number of metastable states is given by

$$
\overline{N_{M S}}=\overline{\operatorname{Tr}_{S_{i}} \prod_{i} \theta\left(\sum_{j \neq i} J_{i j} S_{i} S_{j}\right)}
$$

The term $\theta$ is the Heaviside function and is only nonzero if every spin $S_{i}$ is aligned with its local field $h_{i}=\sum_{j \neq i} J_{i j} S_{j} i$.e. when $h_{i} S_{i}>0$. The average number of metastable states at average energy $E$ per spin is given by

$$
\overline{N_{M S}(E)}=\overline{\operatorname{Tr}_{S_{i}}\left[\prod_{i} \theta\left(\sum_{j \neq i} J_{i j} S_{i} S_{j}\right)\right] \delta\left(E N+\frac{1}{2} \sum_{i j} J_{i j} S_{i} S_{j}\right)}
$$

To proceed we make the gauge transformation $\mathcal{O}_{i j} \rightarrow \mathcal{O}_{i j} S_{i} S_{j}=\mathcal{O}_{i j}^{\prime}$, it is easy to see that $\mathcal{O}^{\prime}$ is also in $O(N)$. One may therefore write

$$
\overline{N_{M S}(E)}=2^{N} \overline{\left[\prod_{i} \theta\left(\sum_{j \neq i} J_{i j}^{\prime}\right)\right] \delta\left(E N+\frac{1}{2} \sum_{i j} J_{i j}^{\prime}\right)}
$$

where $J^{\prime}=\mathcal{O}^{\prime T} \Lambda \mathcal{O}^{\prime}$. Following the standard method [2] we use the identity

$$
\theta(x)=\int_{0}^{\infty} d x \int_{-\infty}^{\infty} \frac{d \lambda}{2 \pi} \exp (i \lambda x)
$$

We thus obtain

$$
\overline{N_{M S}}=2^{N} \int \frac{d \mu}{2 \pi} \frac{d x_{i} d \lambda_{i}}{2 \pi} \overline{\exp \left(i \sum_{i j} J_{i j}^{\prime}\left(\lambda_{i}+\frac{\mu}{2}\right)-i \sum_{i} J_{i i}^{\prime} \lambda_{i}-i \sum_{i} \lambda_{i} x_{i}+i N \mu E\right)}
$$

To simplify the algebra we make the change of variables $\lambda_{i} \rightarrow \lambda_{i}-\frac{\mu}{2}$. Following [5, 13] we now consider the term

$$
\Omega=\overline{\exp \left(i \sum_{i j} J_{i j} \lambda_{i}\right)}=\overline{\exp \left(\frac{i}{2} \operatorname{Tr} J(M-2 L)\right)}
$$


Where in vectorial notation $M=\lambda \mathbf{u}^{T}+\mathbf{u} \lambda^{T}$ with $\mathbf{u}_{i}=1, \lambda_{i}=\lambda_{i}$ and $L_{i j}=\left(\lambda_{i}-\mu / 2\right) \delta_{i j}$. We note here that in order to eliminate the diagonal term in Eq. (12) the matrix $L$ has appeared, and this term must be properly accounted for to obtain the correct result. The averaging over the $O(N)$ disorder (the Haar measure) in this problem can be carried out by using the results of 12 for $U(N)$ integration and adapting them to the $O(N)$ case [7]. Recently a simple replica method was also used to derive these results [8]. The results [12, 7, 8] give for an arbitrary symmetric matrix $M$ and $J=\mathcal{O}^{\mathcal{T}} \Lambda \mathcal{O}$ that

$$
\overline{\exp \left[\frac{1}{2} \operatorname{Tr} M J\right]}=\exp \left[\frac{N}{2} \operatorname{Tr} G\left(\frac{M}{N}\right)+\text { nonextensive terms }\right]
$$

where the overline indicates the Haar average over $\mathcal{O}$ and to leading order the non extensive terms are of order one. A compact formula for $G$ is [8]

$$
G(z)=\max _{\mu}\left\{\mu z-\int d \lambda \frac{\rho(\lambda)}{\mu-\lambda}-\ln (z)-1\right\}
$$

In the models of interest here $G(z)$ is given by 8

$$
\begin{aligned}
G(z) & =\frac{1}{2}\left[(1+4 z(m+z))^{\frac{1}{2}}+m \ln \left((1+4 z(m+z))^{\frac{1}{2}}+2 z+m\right)\right. \\
& \left.-\ln \left((1+4 z(m+z))^{\frac{1}{2}}+1+2 m z\right)-m \ln (m+1)-1-\ln (2)\right] \\
G(z) & =-\alpha \ln (1-z) \quad(\mathrm{FH}) \\
G(z) & =\alpha \ln (1+z)(\mathrm{AFH})
\end{aligned}
$$

Following the results of $\llbracket 7,12,8 \rrbracket$ one obtains

$$
\Omega=\exp \left(\frac{N}{2} \operatorname{Tr} G\left(i \frac{M-2 L}{N}\right)\right)
$$

Given the form of the matrix $M$, the only non zero eigenvalues of $M$ are in the vector subspace of $\mathbb{R}^{N}$ spanned by $\lambda$ and $\mathbf{u}$ [5]. The two non zero eigenvalues are

$$
\mu_{ \pm}=\lambda \cdot \mathbf{u} \pm|\lambda||\mathbf{u}|
$$

We define the order parameters $z$ and $v$ by

$$
\begin{aligned}
& z=\frac{1}{N} \sum_{i} \lambda_{i} \\
& v=\frac{1}{N} \sum_{i} \lambda_{i}^{2}
\end{aligned}
$$

Hence the matrix $\tilde{M}=M / N$ has eigenvalues $z+\sqrt{v}$ and $z-\sqrt{v}$ which are of order 1 and the other $N-2$ eigenvalues are zero. We now consider the evaluation of the term $\operatorname{Tr} G\left(i \frac{M-2 L}{N}\right)$, Taylor expanding one has

$$
\operatorname{Tr} G\left(i \frac{M-2 L}{N}\right)=\sum_{n=0}^{\infty} \frac{i^{n}}{n !} G^{(n)}(0) \operatorname{Tr} \frac{(M-2 L)^{n}}{N^{n}}
$$

We note that for finite values of the $\lambda_{i}$ that to leading order $\operatorname{Tr} M^{p}=C(p) N^{p}$ and that $\operatorname{Tr} L^{p}=D(p) N$. Also for any product $P_{p}(M, L)$ of the $M$ and $L$ containing $p$ factors 
one has that for $p \geq 1 \operatorname{Tr} P_{p}(M, L)<N^{p+\epsilon}$ when $N$ is large for any small positive $\epsilon$. The dominant terms of this form are when $P_{p}(M, L)=M^{p}$. To see this, consider a product $P_{p}(M, L)$ with at least one $L$ occurring, we can thus write, exploiting the Cauchy-Schwartz inequality

$$
\begin{aligned}
\operatorname{Tr} P_{p}(M, L) & =\operatorname{Tr} L P_{p-1}(M, L) \\
& \leq\left(\operatorname{Tr} L^{2}\right)^{\frac{1}{2}}\left(\operatorname{Tr} P_{p-1}(M, L)^{2}\right)^{\frac{1}{2}} \\
& \leq \text { Const } N^{\frac{1}{2}} \times N^{p+\epsilon-1}=\text { Const } N^{p+\epsilon-\frac{1}{2}}
\end{aligned}
$$

Hence for $p \geq 2$ any product containing at least one $L$ is such that $\operatorname{Tr} P_{p}(M, L) / N^{p} \leq$ Const $N^{\epsilon-\frac{1}{2}} \rightarrow 0$. The only term containing $L$ which survives the thermodynamic limit is that in the linear term of the Taylor expansion (23). Putting all this together for large $N$ yields

$$
\Omega=\exp \left(N\left(\frac{1}{2} G(i z+i \sqrt{v})+\frac{1}{2} G(i z-i \sqrt{v})-i\left(z-\frac{\mu}{2}\right) G^{\prime}(0)\right)\right)
$$

It is easy to show that $G^{\prime}(0)=\int d \lambda \lambda \rho(\lambda)$, and hence in the ROM $G^{\prime}(0)=2 \alpha-1$, which is zero when $\alpha=1 / 2$ explaining why the diagonal term mentioned above was unimportant in the calculations of [5, 13]. It is easy to see heuristically the origin of this term, as it comes from the term $D=\sum_{i} J_{i i}^{\prime} \lambda_{i}$ in Eq.([12), the results of the above calculation is to show that in the large $N$ limit $D$ can be written as $D \approx\left(\frac{1}{N} \sum_{i} J_{i i}^{\prime}\right)\left(\sum_{i} \lambda_{i}\right)=\frac{1}{N} \operatorname{Tr}(J)\left(\sum_{i} \lambda_{i}\right)$.

Introducing a delta function representation for the order parameters, the $x_{i}$ and $\lambda_{i}$ and $\mu$ integrals may be done yielding

$$
\overline{N_{M S}(E)}=\frac{N^{\frac{3}{2}}}{16 \pi^{2}} \int d z d v d s d t\left(\frac{8 \pi}{t}\right)^{\frac{1}{2}} \exp (N A[z, v, s, t, E])
$$

where

$$
\begin{aligned}
A[z, v, s, t, E] & =\frac{1}{2}(G(z+i \sqrt{v})+G(z-i \sqrt{v}))-z G^{\prime}(0)-s z+\frac{v t}{2} \\
& +B\left(\frac{s}{\sqrt{t}}\right)+\frac{2}{t}\left(E+\frac{s}{2}+\frac{t z}{2}+\frac{1}{2} G^{\prime}(0)\right)^{2}
\end{aligned}
$$

where

$$
\begin{aligned}
B(u) & =\ln \left(\sqrt{\frac{2}{\pi}} \int_{-u}^{\infty} d x \exp \left(-\frac{x^{2}}{2}\right)\right) \\
& =\ln \left(1+\operatorname{erf}\left(\frac{u}{\sqrt{2}}\right)\right)
\end{aligned}
$$

and anticipating a real action we have made the transformation $z \rightarrow-i z$ The average energy $E^{*}$ of the metastable states is then given by

$$
E^{*}=-\frac{s}{2}-\frac{t z}{2}-\frac{1}{2} G^{\prime}(0)
$$

at the saddle point of the reduced action

$$
A[z, v, s, t]=\frac{1}{2}(G(z+i \sqrt{v})+G(z-i \sqrt{v}))-z G^{\prime}(0)-s z+\frac{v t}{2}+B\left(\frac{s}{\sqrt{t}}\right)(30
$$


The total average number of metastable states is given by

$$
\overline{N_{M S}}=N \int d E \overline{N_{M S}(E)}
$$

which gives

$$
\overline{N_{M S}}=\frac{N^{2}}{8 \pi^{2}} \int d z d v d s d t \exp (N A[z, v, s, t])
$$

We have therefore to leading order

$$
S^{*}=\frac{\ln \left(\overline{N_{M S}}\right)}{N}=\operatorname{extr}_{z, v, s, t} A[z, v, s, t]-\frac{\ln (2)}{N}-\frac{1}{2} \frac{\ln (\operatorname{det} \mathcal{H})}{N}+O(1 / N)(
$$

where $\mathcal{H}$ is the Hessian of $A$ at the saddle point and the term $O(1 / N)$ comes from the nonextensive terms arising in the $O(N)$ disorder averaging. The fact that the leading order correction is $O(1 / N)$ means that $S^{*}$ can be evaluated by exact enumeration for quite small system sizes when the above finite size scaling is taken into account.

The extremization of this action (with four order parameters) seems quite complicated and has a similar structure to the saddle point encountered in the calculation of the average number of metastable states in the periodic glass model studied in [6] the Hopfield model [4, 3] and the ROM at $\alpha=1 / 2$ [5, 13]. The saddle point equations giving $s$ and $t$ are

$$
\begin{aligned}
& s=\frac{1}{2} G^{\prime}(z+i \sqrt{v})+\frac{1}{2} G^{\prime}(z-i \sqrt{v})-G^{\prime}(0) \\
& t=-\frac{i}{2 \sqrt{v}}\left(G^{\prime}(z+i \sqrt{v})-G^{\prime}(z-i \sqrt{v})\right)
\end{aligned}
$$

The remaining saddle point equations must be solved numerically. Given the definition of the order parameter $v$ we look for solutions to the saddle point equations with positive $v$. We shall see that the solutions we find with this prescription agree perfectly with the results of exact enumeration of small systems where we can calculate both $S^{*}$ the entropy of metastable states and $E^{*}$ the average energy of these states.

The calculated values of $S^{*}(\alpha)$ for the ROM and ferromagnetic Hopfield models along with $S^{*}(1-\alpha)$ for the anti-ferromagnetic Hopfield model are shown in Fig. (11). As the ROM can be regarded as an anti-ferromagnetic Hopfield model with $1-\alpha$ orthonormal patterns or a ferromagnetic Hopfield model with $\alpha$ orthonormal patterns, this comparison is natural. In the limit $\alpha \rightarrow 1$ we see in Fig. (11) that $S_{\mathrm{ROM}}^{*}(\alpha) \rightarrow$ $S_{\mathrm{AFH}}^{*}(1-\alpha)$ and that in these two cases $S^{*} \rightarrow \ln (2)$. However $S_{F H}^{*} \approx 0.131486$. Hence, for the ROM, as $\alpha \rightarrow 1$ there is a small fraction $(1-\alpha)$ of repulsive patterns to be avoided to minimize the energy and the fact that they are strictly orthonormal or statistically orthonormal does not change the behavior of $S^{*}$ drastically with respect to the AFH. This result can be seen analytically as follows. Writing $\alpha^{\prime}=1-\alpha$, then near $\alpha=1$, for the ROM

$$
G(z)=z-\alpha^{\prime} \ln (1+2 z)+O\left(\alpha^{\prime 2}\right)
$$

In the metastable state calculations here the term linear in $z$ of $G(z)$ disappears from the calculation. The remaining term is just (up to a rescaling of the energy that will not 


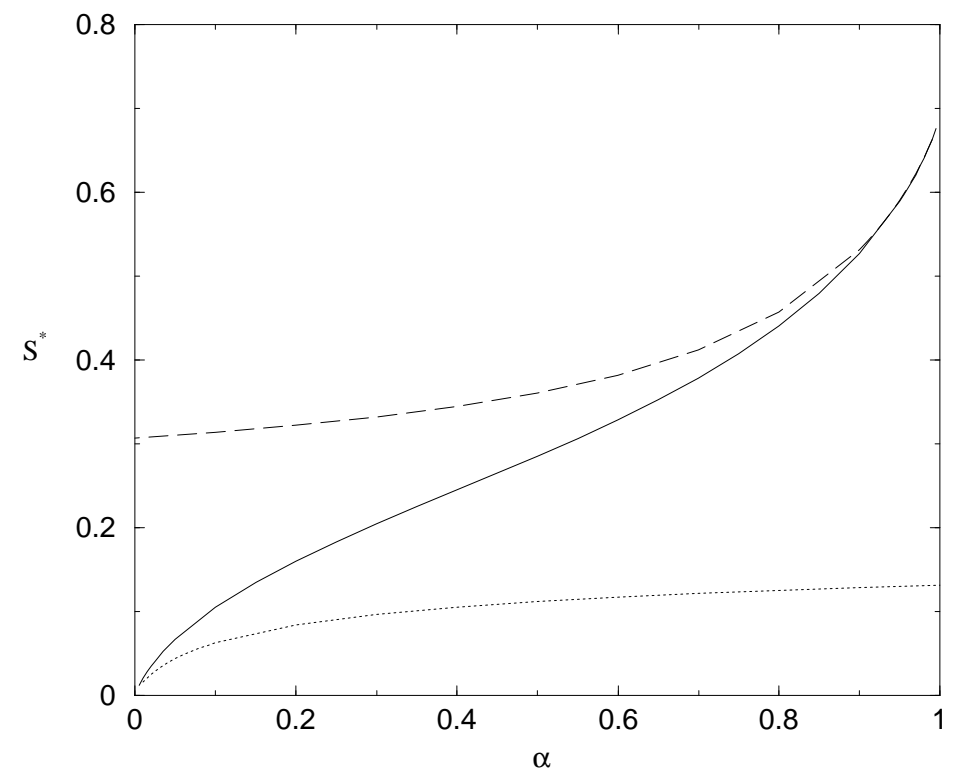

Figure 1. The entropy of metastable states per spin for (i) ROM (full line) (ii) FH with $p=\alpha N$ patterns (dotted line) and (iii) AFH with $p=(1-\alpha) N$ patterns (dashed line)

affect the number of metastable states) the term one has for the AFH with $\alpha^{\prime}$ patterns. Thus explaining the convergence of $S^{*}(\alpha)$ for the ROM with $S^{*}(1-\alpha)$ for the AFH near $\alpha=1$. One may further show that in these two cases as $\alpha \rightarrow 1$ one has

$$
S^{*}(\alpha) \approx \ln (2)-\frac{\alpha^{\prime}}{2}\left[\ln \left(\frac{2}{e \alpha^{\prime}} \ln \left(\frac{1}{\alpha^{\prime}}\right)\right)+\frac{1}{\ln \left(\frac{1}{\alpha^{\prime}}\right)}\right]
$$

This asymptotic formula agrees well with the numerically calculated value up to $\alpha^{\prime}=0.1$.

In the limit $\alpha \rightarrow 0$ we also see that $S_{\mathrm{ROM}}^{*}\left(0^{+}\right)=0$ and $S_{\mathrm{FH}}^{*}\left(0^{+}\right)=0$ but $S_{\mathrm{AFH}}^{*}(1) \approx 0.306983$. However $S_{\mathrm{ROM}}^{*}$ and $S_{\mathrm{FH}}^{*}$ remain different as $\alpha \rightarrow 0$. Hence when the attractive patterns are strictly orthonormal then there are more metastable states than if the patterns are only statistically orthonormal. In the $\mathrm{FH}$ model in the limit $\alpha \rightarrow 0$ it was shown [3] that $S^{*}(\alpha) \approx \frac{1}{\alpha}[\ln (2 / \pi \alpha)-1]$. In the same limit in the ROM the asymptotic behavior is rather singular and we have not yet found the corresponding asymptotic behavior.

To summarize we have the inequality $S_{\mathrm{FH}}^{*}(\alpha) \leq S_{\mathrm{ROM}}^{*}(\alpha) \leq S_{\mathrm{AFH}}^{*}(1-\alpha)$. Hence in the case of ferromagnetic Hopfield models strict pattern orthonormality increases the number of metastable states but in the anti-ferromagnetic Hopfield model it decreases the number of metastable states. Let us note here that at a fixed pattern number the $\mathrm{ROM}$ and $\mathrm{AFH}$ have more metastable states than the FH. This is in accordance with the observation that the ROM and AFH have a structural glass transition whereas the FH has a continuous spin glass transition. 


\section{Numerical Simulations}

To verify our results we have carried out exact numerical enumeration of $N_{M S}$ on small systems of size between 10 and 30 spins. Using the finite size scaling predicted by Eq. (33) we find excellent agreement between the calculations presented here even for the relatively small system sizes examined.

Measured in the simulations were $\overline{N_{M S}}, \overline{\ln \left(N_{M S}\right)}$ and $E^{*}$ the average value of the energy per spin of the metastable states. The numerical results confirm to high precision that $\ln \left(\overline{N_{M S}}\right)=\overline{\ln \left(N_{M S}\right)}$, thus confirming that the total entropy on metastable states is self averaging and justifying our annealed calculation. Averaging was carried out over up to $2^{35-N}$ samples for the systems size of size $N$, with $N$ between 10 and 30 . Both the annealed total entropy of metastable states $N S^{*}=\ln \left(\overline{N_{M S}}\right)$ and the quenched total entropy $N S_{q}^{*} \overline{\ln \left(N_{M S}\right)}$ were plotted as function of $N$ and were found to be very close to straight lines for systems of size greater than 10 . The value of $S^{*}$ was then determined by a linear fit. The average energy per spin over all metastable states and samples, corresponding to the annealed calculations carried out here,

$$
E^{*}=\frac{\int d E E \overline{N_{M S}(E)}}{\int d E \overline{N_{M S}(E)}}
$$

was calculated from the simulations of the systems of size 20 (to have good statistics). As an additional check the quenched average energy per spin of the metastable states

$$
E_{q}^{*}=\overline{\left(\frac{\int d E E N_{M S}(E)}{\int d E N_{M S}(E)}\right)}
$$

was also calculated. We note that if annealed approximation is exact then we should find $E^{*}=E_{q}^{*}$.

The results for $S^{*}$ estimated from the annealed average $\ln \left(\overline{N_{M S}}\right)$ and the quenched average $\overline{\ln \left(N_{M S}\right)}$ in the $\mathrm{AFH}$ are shown in Fig. (2) against the calculated value. We see again that the agreement is excellent. The difference between the annealed and quenched averages are very small showing the validity of the annealed approximation. Note that by Jensen's inequality the the annealed average should be greater than the quenched one. Similarly the numerically estimated values for $E^{*}$ and $E_{q}^{*}$ are within the error bars of the simulation and also in excellent agreement with the calculated value of $E^{*}$.

For the ROM, in each system of size $N, N \alpha$ eigenvectors were chosen to have eigenvalue 1 and the remaining to have eigenvalue -1 . The extrapolated values of $S^{*}$ and $S_{q}^{*}$ are shown in Fig.(3) along with the energy $E_{q}^{*}$ obtained from samples of size $N=30$. We see that again that agreement with the analytical calculations is excellent and that the extrapolated values of $S^{*}$ and $S_{q}^{*}$ coincide.

Via the exact numerical enumeration were also computed the annealed $N S^{*}(E)=$ $\ln \left(\overline{N_{M S}(E)}\right)$ and quenched $N S_{q}^{*}(E)=\overline{\ln \left(N_{M S}(E)\right)}$ entropy of the metastable states of 


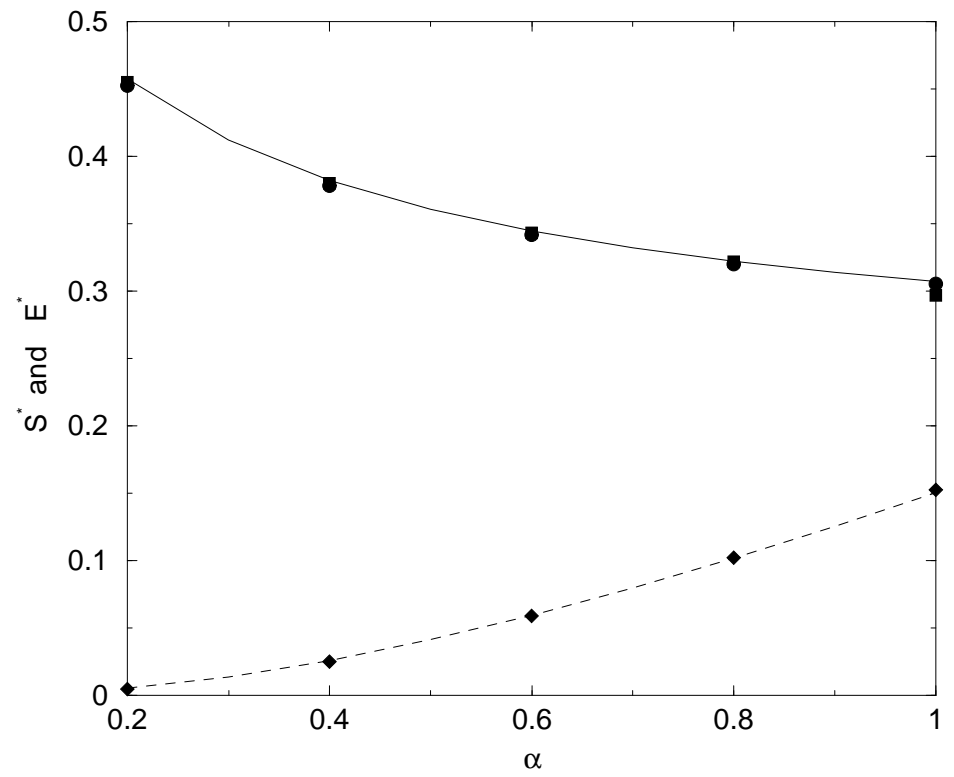

Figure 2. The annealed $\left(S^{*}\right)$ (circles) and quenched $\left(S_{q}^{*}\right)$ (squares) entropy of metastable states for the anti-ferromagnetic Hopfield model measured from the simulations along with the calculated value (solid line). Also shown is the average values of the energy per spin of the metastable states measured by the simulations (diamonds) along with the calculated value $E^{*}$.

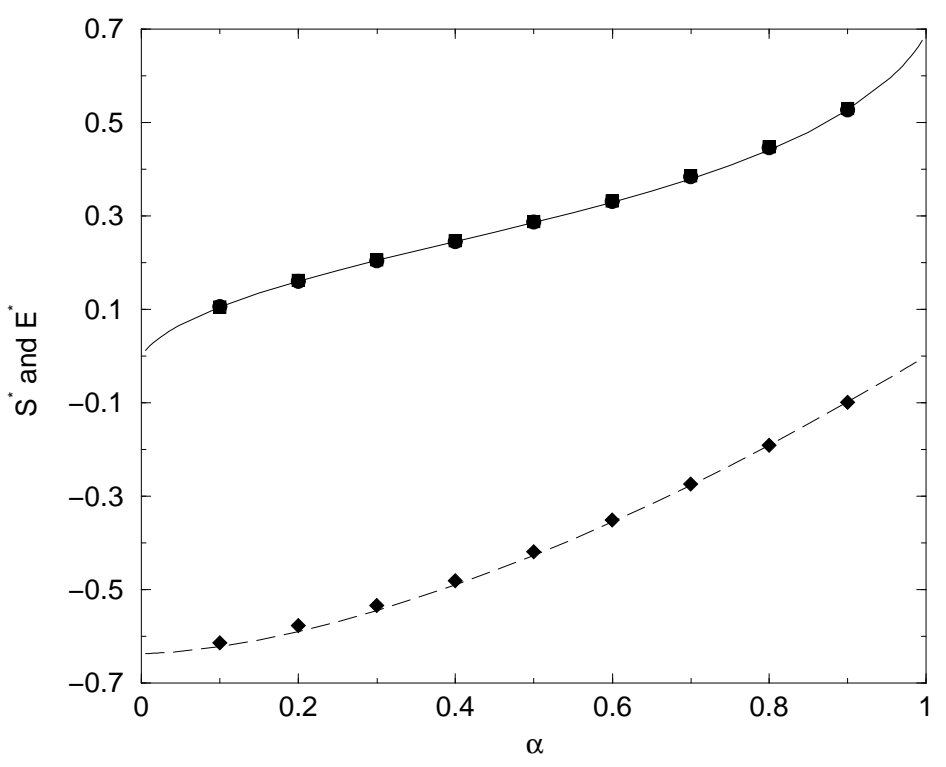

Figure 3. As in Fig. (2), but for the generalized ROM. For the average energy, the errors where computed and are always less than the size of the symbols. 


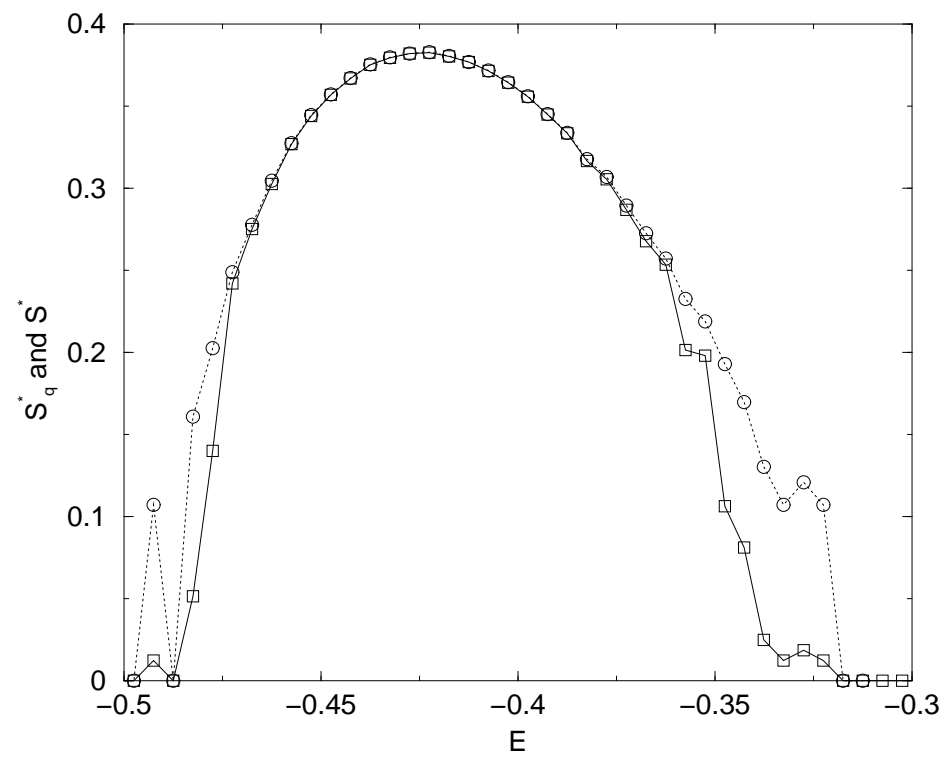

Figure 4. The annealed $\left(S^{*}(E)\right)$ (circles, solid line) and quenched $\left(S_{q}^{*}(E)\right)$ (squares, dotted line) entropy of metastable states of energy $E$ per spin for the ROM.

energy E. Numerically, we computed

$$
\overline{N_{M S}\left(E_{i}\right)}=\frac{1}{\# \text { samples }} \sum_{i=1}^{\# \text { samples }} \frac{N_{M S}\left(E_{i}, E_{i}+\Delta E\right)}{\Delta E}
$$

and

$$
\overline{\ln \left(N_{M S}\left(E_{i}\right)\right)}=\frac{1}{\# \text { samples }} \sum_{i=1}^{\# \text { samples }} \ln \left(\frac{N_{M S}\left(E_{i}, E_{i}+\Delta E\right)}{\Delta E}\right)
$$

where $\Delta E=.005$ is the chosen bin size of the discrete energy values $E_{i}$. To avoid divergences when taking the logarithm, we set $\ln \left(N_{M S}(E)\right)=0$ when $N_{M S}(E)$ was zero in a given sample. This procedure should be unimportant in the thermodynamic limit as it concerns a nonextensive number of metastable states. The results for the ROM with $\alpha=1 / 2$ are shown in Fig.(4) for an averaging over 32 samples of size 30. We see clearly, for a substantial region around the most probable energy, the annealed and quenched entropies coincide i.e. $S^{*}(E)=S_{q}^{*}(E)$. For values of the entropy smaller than 0.3 , the two curves depart from each other, with $S_{q}^{*}$ always smaller than $S^{*}$ as it should be. However, the small number of metastable states considered for these energies and size makes it impossible to draw any conclusion about the thermodynamic limit. In particular one would expect that the two entropies should collapse for all energies above some energy threshold $E_{0}$ below $E^{*}$. This is not the case for our data indicating strong finite size effects at the edges of the energy spectrum of the metastable states, as one should expect. 


\section{Conclusions}

We have shown that in the class of ROMs considered here that there is always an exponentially large number of metastable states which increases as a function of $\alpha$. The ROM can be viewed as a ferromagnetic Hopfield model with $\alpha$ strictly orthonormal patterns or and anti-ferromagnetic Hopfield model with $1-\alpha$ strictly orthonormal patterns. Comparison with the corresponding Hopfield models shows that the orthonormality of the patterns increases the number of metastable states in the ferromagnetic case but decreases this number in the anti-ferromagnetic case. If one considers a ferromagnetic Hopfield model with all patterns parallel then there are clearly only two metastable states (all spins aligned or anti-aligned with this pattern). In the anti-ferromagnetic Hopfield model if all the patterns are parallel then there are more metastable states as it is easier to be orthogonal to a single pattern than several. This reasoning in these extreme cases is compatible with the results found here.

Finally the numerical simulations carried out here, though for small system sizes, show remarkable agreement with the analytic calculations carried out here. This is extremely important as the structure of the saddle point equations is so complicated that one needs some confirmation that one has found the good saddle point. Furthermore it suggests that rather than doing Monte Carlo simulations for systems exhibiting a dynamical transition, where even small size systems will stay out of equilibrium, it may be more useful to carry out exact enumeration on these small system sizes to calculate thermodynamic quantities.

\section{Acknowledgments}

During this work we have benefited from many interesting discussions with A.J. Bray and S.N. Majumdar.

\section{References}

[1] K. Binder and A.P. Young, Rev. Mod. Phys. 58, 801 (1986); M. Mézard, G. Parisi and M.A. Virasoro, Spin Glass Theory and Beyond (World Scientific, Singapore 1987); K.H. Fischer and J.A. Hertz, Spin Glasses (Cambridge University Press, Cambridge 1991); V. Dotsenko, Introduction to the Replica Theory of Disordered Statistical Systems, (Cambridge University Press, Cambridge 2000)

[2] F. Tanaka and S.F. Edwards J. Phys. F 13, 2769 (1980); A.J. Bray and M.A. Moore, J. Phys. C. 13, L469 (1980); A.J. Bray and M.A. Moore, J. Phys. C. 14, 1313 (1981); C. de Dominicis, M. Gabay, T. Garel and H. Orland J. Physique 41, 923 (1980); S.A. Roberts J. Phys. C. 14, 3015 (1981); D.S. Dean J. Phys. A. 27, L899 (1994); V.M.de Oliveira and J.F. Fontanari J. Phys. A. 30, 8445 (1997)

[3] E. Gardner J. Phys. A 19, L1047 (1986).

[4] A Treves and D.J. Amit J. Phys. A 21, 3155 (1988).

[5] G. Parisi and M. Potters J. Phys. A 28, 5267 (1995).

[6] P. Chandra, L. B. Ioffe, and D. Sherrington Phys. Rev. B 58, 14669(R) (1998). 
[7] E. Marinari, G. Parisi and F. Ritort J. Phys. A 27, 7615 (1994); E. Marinari, G. Parisi and F. Ritort J. Phys. A 27, 7647 (1994).

[8] R. Cherrier, D.S. Dean and A. Lefèvre, cond-mat 0211695.

[9] E. Gardner Nucl. Phys. B. 257, 747 (1985); H. Rieger, Phys. Rev. B 46, 14655 (1992); A. Crisanti and H. J. Sommers Z, Phys. B 87, 341 (1992); A. Crisanti and H. J. Sommers J. Phys. I (France) 5, 805 (1995).

[10] D.S. Dean and F. Ritort Phys. Rev. B. 65, 224209, (2002).

[11] A. De Martino and M. Marsili J. Phys. A 34, 2525 (2001).

[12] C. Itzykson and J.B. Zuber, J. Math. Phys. 21, 411 (1980)

[13] M. Degli Esposti, C. Giardina and S. Graffi, cond-mat 0207681. 